Western University

Scholarship@Western

Human Environments Analysis Lab (HEAL)

$2-2016$

Factors that influence excessive gestational weight gain: Moving beyond assessment and counselling

Emily E, Campbell

Paula D N Dworatzek

Debbie Penava

Barbra De Vrijer

Jason A. Gilliland

See next page for additional authors

Follow this and additional works at: https://ir.lib.uwo.ca/healpub 


\section{Authors}

Emily E, Campbell; Paula D N Dworatzek; Debbie Penava; Barbra De Vrijer; Jason A. Gilliland; June I. Matthews; and Jamie A Seabrook 


\title{
Factors that influence excessive gestational weight gain: moving beyond assessment and counselling
}

\author{
Emily E. Campbell, Paula D. N. Dworatzek, Debbie Penava, Barbra de Vrijer, \\ Jason Gilliland, June I. Matthews \& Jamie A. Seabrook
}

To cite this article: Emily E. Campbell, Paula D. N. Dworatzek, Debbie Penava, Barbra de Vrijer, Jason Gilliland, June I. Matthews \& Jamie A. Seabrook (2016) Factors that influence excessive gestational weight gain: moving beyond assessment and counselling, The Journal of Maternal-Fetal \& Neonatal Medicine, 29:21, 3527-3531, DOI: 10.3109/14767058.2015.1137894

To link to this article: https://doi.org/10.3109/14767058.2015.1137894

Accepted author version posted online: 08

Jan 2016.

Published online: 10 Feb 2016.

Submit your article to this journal $匚$

山ll Article views: 572

Q View related articles ¿ك

View Crossmark data ¿

4

Citing articles: 5 View citing articles $\sqsubset$ 


\title{
Factors that influence excessive gestational weight gain: moving beyond assessment and counselling
}

\author{
Emily E. Campbell ${ }^{1}$, Paula D. N. Dworatzek ${ }^{1,2}$, Debbie Penava ${ }^{3,4}$, Barbra de Vrijer ${ }^{3,4}$, Jason Gilliland ${ }^{3,5,6,7,8}$, \\ June I. Matthews ${ }^{1}$, and Jamie A. Seabrook ${ }^{1,3,5,6}$ \\ ${ }^{1}$ Division of Food and Nutritional Sciences, Brescia University College, Western University, London, ON, Canada, ${ }^{2}$ Schulich Interfaculty Program in \\ Public Health, Western University, London, ON, Canada, ${ }^{3}$ Children's Health Research Institute, London, ON, Canada, ${ }^{4}$ Department of Obstetrics and \\ Gynaecology, Western University, London, ON, Canada, ${ }^{5}$ Department of Paediatrics, Western University, London, ON, Canada, ${ }^{6}$ Lawson Health \\ Research Institute, London, ON, Canada, ${ }^{7}$ Department of Geography, Western University, London, ON, Canada, and ${ }^{8}$ School of Health Studies, \\ Western University, London, ON, Canada
}

\begin{abstract}
One in four Canadian adults is obese, and more women are entering pregnancy with a higher body mass index (BMI) than in the past. Pregnant women who are overweight or obese have a higher risk of pregnancy-related complications than women of normal weight. Gestational weight gain (GWG) is also associated with childhood obesity. Although the factors influencing weight gain during pregnancy are multifaceted, little is known about the social inequality of GWG. This review will address some of the socioeconomic factors and maternal characteristics influencing weight gain and the impact that excessive GWG has on health outcomes such as post-partum weight retention. The effects of an overweight or obese pre-pregnancy BMI on GWG and neonatal outcomes will also be addressed. The timing of weight gain is also important, as recommendations now include trimester-specific guidelines. While not conclusive, preliminary evidence suggests that excessive weight gain during the first trimester is most detrimental.
\end{abstract}

\section{Keywords}

Gestational weight gain, pregnancy, social class, socioeconomic status (SES), weight gain timing

\section{History}

Received 21 October 2015

Accepted 30 December 2015

Published online 4 February 2016

\section{Introduction}

Recent estimates suggest that $25 \%$ of Canadian adults are obese [1]. Increased healthcare costs have accompanied the rise in obesity rates, mostly due to higher risks of obesityrelated co-morbidities such as cardiovascular disease and type 2 diabetes [1]. Accordingly, obesity has become the focus of many public health initiatives.

One important consequence of the obesity epidemic is that many women are entering pregnancy with an elevated body mass index (BMI) [2]. This is problematic because obesity during pregnancy increases health risks for both mother and foetus. Overweight and obese mothers are more likely to experience post-partum weight retention, and infants born to these mothers have a higher probability of childhood obesity [3]. A goal of clinical practice guidelines has been to reduce the number of women who are overweight and obese during the reproductive period through the promotion of a healthful lifestyle before, during and after pregnancy, and to increase physician involvement for those considered a high risk

Address for correspondence: Jamie A. Seabrook, PhD, Division of Food and Nutritional Sciences, Brescia University College at Western University, 1285 Western Road, London, Ontario N6G 1H2, Canada. Tel: +1-519-432-8353(28284). Fax: +1-519-8585137. E-mail: jseabro2@uwo.ca pregnancy [2]. As pregnancy is a critical life stage, it is an opportunity for change and adaptation of healthful behaviours.

\section{Methods}

A search of PubMed, Proquest, PsychInfo and Google Scholar was conducted for full articles available on human studies in English since 2000. Search terms used were: gestational weight gain (GWG) or pregnancy or weight gain timing and socioeconomic status or SES. Titles, key words and abstracts were reviewed to determine relevance. Reference lists of all relevant studies were also examined for any pertinent studies that may have been missed in the review of the aforementioned databases. Given the complexity and challenge of obtaining every original article related to factors influencing excessive GWG, this review seeks to provide an overview of the literature and therefore will not capture all of the current literature.

\section{GWG concerns and recommendations}

It is well-documented that women who gain too much weight during pregnancy have a higher probability of adverse consequences such as excessive body weight, gestational diabetes, hypertension, foetal macrosomia, and premature and 
caesarean delivery [4]. These adverse birth outcomes can be attributed to the influence of pre-pregnancy BMI. Additionally, a recent meta-analysis suggested that between 2000 and 2009, 44.4\% of women gained weight above weight gain guidelines [4]. Maternal BMI has also been found to be positively associated with infant birth weight across all categories of GWG [5]. Interestingly, pre-pregnant women in the underweight and normal BMI categories develop the same complications as women who are overweight at conception, if weight gain exceeds the recommended amount [5]. Furthermore, a retrospective cohort study of $>70000$ women in Ontario, Canada showed that overweight/obese BMI status, combined with a diagnosis of depression, was associated with the most adverse birth outcomes [6].

The 2009 Institute of Medicine (IOM) recommendations for GWG and the 2010 Society of Obstetricians and Gynaecologists of Canada (SOGC) Clinical Practice Guidelines provide guidance to assist women in gaining an appropriate amount of weight to minimise the risk of adverse birth outcomes and post-partum weight retention, increase the likelihood of a healthy birth weight, and in turn, avoid the risk of childhood overweight and obesity [2,7]. The "Developmental Origins of Adult Disease" concept suggests that babies born after being in an adverse in utero environment have an increased risk of metabolic syndrome [8]. As a result, it is important to find a balance in GWG guidelines to prevent small-for-gestational-age (SGA) infants when there is low GWG or weight loss, as well as large-for-gestational-age (LGA) infants when excessive GWG (eGWG) occurs [6].

Women of all BMI categories appear to be gaining excessive weight during pregnancy. However, eGWG is particularly problematic for women whose pre-pregnancy BMI is in the overweight or obese categories, since these women are already at higher risk for pregnancy complications [5]. Efforts must therefore focus on preventing eGWG in overweight and obese women, as these women are more likely to have excessive weight gain during pregnancy due to the narrow IOM recommendations for these BMI categories [7]. Infants born to mothers with eGWG have a higher risk of macrosomia, which is in turn associated with obesity, impaired glucose tolerance and infant mortality [3].

\section{The impact of eGWG on maternal and child health}

Studies have found that eGWG, independent of pre-pregnancy obesity, can have short- and long-term consequences for maternal and child health $[9,10]$. eGWG is associated with a greater likelihood of a caesarean section, delivering a LGA neonate, and post-partum weight retention [3,4]. Savitz et al. [11] investigated the association between GWG and birth outcomes across all BMI categories and found that eGWG among overweight and obese women was more strongly associated with preterm birth compared to normal weight women. This is consistent with recently published work suggesting that overweight and obese women may struggle to stay within the IOM recommendations for GWG, and that eGWG in overweight and obese women increases the risk for weight retention post-partum [10,12]. Studying the Danish National Birth Cohort, Nohr et al. [12] showed that pregnancy complications such as hypertension, gestational diabetes, pre-eclampsia and caesarean birth were more common in overweight and obese women.

Given the relationship between eGWG and short- and long-term negative health outcomes for mothers and infants, more resources are needed to ensure that pregnant women understand the importance of gaining weight within IOM recommendations, and that they have the necessary support to achieve these recommendations [2]. Healthcare professionals that have the most frequent medical contact with women of childbearing age (e.g. primary care physicians, obstetricians/gynaecologists, registered dietitians) are best suited for this task. Preliminary evidence suggests that patient-centred advice from a prenatal care provider will increase patient acceptance and adherence to recommendations [13].

\section{Socioeconomic factors influencing GWG}

Although the association between eGWG and adverse pregnancy and birth outcomes are well-established, few studies have investigated the extent to which socioeconomic factors influence GWG. One exception is a recent study investigating the influence of childhood SES as a predictor of eGWG and obesity in adulthood [14]. Using data from the National Longitudinal Survey of Youth (1970-2010), childhood socioeconomic disadvantage was not associated with eGWG but was associated with obesity, suggesting that eGWG does not mediate the relationship between childhood SES and obesity in adulthood.

Income is an important indicator of SES because it influences many factors that also predict health, such as the ability to purchase nutritious food, safe and affordable housing, adequate and appropriate transportation, and college or university education [15]. Women with low income have an increased risk for both inadequate and excessive GWG, in part because many lack an understanding about the importance of a healthy diet during pregnancy and have limited access to healthy food, often leading to a higher consumption of energy-dense food [15]. Even when there is access to nutritious food, the cost makes it difficult for a low income family to purchase it. In fact, food price is the primary determinant of food choice among families living in poverty, which often results in selecting foods high in fat and sugar [16].

Economically disadvantaged women are also more likely to live in neighbourhoods characterised by socioeconomic disadvantage than are women who are financially secure. These disadvantaged neighbourhoods tend to have limited opportunities for employment; inadequate access to healthcare, grocery stores and transportation; and experience higher rates of violence and crime [17]. Living in communities with high socioeconomic disadvantage is associated with higher BMI, controlling for age, race, individual SES, smoking, physical activity, stress and social support [18]. As a result, those of lower SES who experience food insecurity may be more likely to have eGWG contributing to adverse birth outcomes. Furthermore, women with college or university education who reside in disadvantaged neighbourhoods are more likely to have eGWG during pregnancy compared to women residing in neighbourhoods with medium or high 
SES [19]. This supports the need for improved preconception and prenatal care to decrease eGWG in low SES areas.

When considering the effect of rural versus urban living on GWG, women living in rural communities have more limited access to healthcare services, forcing some pregnant women to travel long distances to access healthcare [20]. One study found that normal and overweight women living in rural areas were at a higher risk for weight changes above or below the IOM recommendations than those living in urban areas because the former have limited medical contact and access to grocery stores during pregnancy [20]. Additionally, eGWG was more common in overweight rural-dwelling women, further suggesting that improvements in access to rural healthcare, physical activity, and healthy foods are necessary for overweight and obese women in these areas if significant improvements in health status are to be achieved [20].

Research on the association between SES and GWG has been limited due to the lack of nationally representative samples that include all socioeconomic groups, thus not allowing for adequate comparisons for GWG across the socioeconomic strata. As well, SES tends to be treated as a confounding factor amongst a host of other risk factors, including but not limited to parity, pre-pregnancy weight, gestational diabetes status, predicting GWG [21]. Consequently, the extent to which SES is driving GWG through more proximate risk factors, such as variation in diet quality, and access to food and exercise between women of lower and higher SES should be investigated further.

There is currently limited research about social inequalities in GWG, although the studies that have been conducted suggest that social factors such as income, neighbourhood advantage/disadvantage, and rural/urban dwelling all influence the amount of weight gain during pregnancy, and that neighbourhood disadvantage may have a greater influence than education at predicting weight gain $[15,20]$. However, more research assessing the direct influence of educational attainment on GWG is necessary before any clear conclusions can be drawn. Nonetheless, strategies that investigate methods to alleviate SES disadvantage, promote GWG within recommendations, and improve access to services are imperative. These strategies go hand in hand with current initiatives such as the Canadian Prenatal Nutrition Programme, which seeks to reach pregnant women living in conditions of risk that are known to increase the likelihood of unfavourable outcomes for themselves and their infants; and the Healthy Babies Healthy Children programme in Ontario, which seeks to help children get a healthy start in life. Given that eGWG predisposes both mother and child for obesity development, a healthy maternal pre-pregnancy BMI and lower rates of eGWG have the potential to improve both short- and longterm maternal and child health outcomes, promote healthy weight gain over time, and reduce overall rates of maternal and childhood obesity.

\section{The influence of the timing of weight gain in pregnancy}

When the IOM updated the recommendations for weight gain during pregnancy in 2009, trimester-specific recommendations were highlighted and, during the second and third trimester, weight gain recommendations were stratified by BMI category [2].

Although eGWG is associated with perinatal health risks, the specific trimester where eGWG has the largest impact on health remains unclear $[10,22,23]$. In a prospective study of 3015 births, eGWG in all trimesters decreased the odds of delivering a SGA infant, and increased the odds of delivering a LGA infant [2,24]. Margerison-Zilko et al. [24] found that each kilogram of first trimester GWG above the IOM recommendations was significantly associated with an increase in birth weight depending on gestational age in all BMI categories (OR: 1.6, 95\% CI: 1.39-1.82), suggesting that early eGWG may influence birth outcomes as well as total GWG. Results from Brazil found that eGWG during the second trimester was associated with increased risk of a LGA infant, and eGWG in the third trimester was associated with preterm and caesarean birth [23]. In a study from Ontario, Davenport et al. [22] found that infants born to women who had eGWG in the first half of pregnancy had a higher birth weight, crown-heel length and excessive body fat compared to women who gained an appropriate amount of weight [22]. The study also found that $55 \%$ of women who had eGWG in the first half of their pregnancy also had excessive total GWG, showing that early eGWG may place women on a trajectory for excessive weight gain throughout their entire pregnancy.

\section{eGWG and post-partum weight retention}

Given the challenge of returning to pre-pregnancy body weight, the likelihood of post-partum weight retention increases as GWG increases [24]. In a study from Prince Edward Island, weight gain $>12 \mathrm{~kg}$ during pregnancy was associated with post-partum weight retention of $>2.5 \mathrm{~kg}$ in underweight, normal and overweight women [25]. Nohr et al. [12] found that women with GWG $>16 \mathrm{~kg}$ retained $>5 \mathrm{~kg}$ of weight post-partum. Additionally, obese women with weight gain within the IOM recommendations were at least $2 \mathrm{~kg}$ lighter than their pre-pregnancy weight, further supporting the need to promote recommendations for GWG. In a study from Nova Scotia, eGWG led to mean postpartum weight retention of 3.7 and $3.5 \mathrm{~kg}$ for overweight and obese women, respectively, regardless of pre-pregnancy BMI [9]. However, research has shown that, compared to a control group, the addition of a dietary and exercise intervention allowed women who were overweight or obese to decrease their weekly weight gain in efforts consistent with IOM recommendations [26]. Furthermore, one study found that the median post-partum weight retention $(2.7 \mathrm{~kg})$ was significantly higher among women in the medium and lower SES categories $(3.2 \mathrm{~kg}$ ) compared to women of higher SES $(1.8 \mathrm{~kg})$, despite no difference reported in pregnancy weight gain [27]. This suggests that interventions targeting SES may also be necessary to prevent post-partum weight retention. Future recommendations addressed to women during/after pregnancy should utilise the strengths of a multidisciplinary team, and include dietary counselling for an appropriate nutritional schedule during pregnancy and a continual support to maintain a healthy weight after pregnancy. 


\section{Relevance to practice}

Dietitians play a key role in the prevention of obesity, including eGWG during pregnancy. Dietitians can be partners in multidisciplinary teams to provide pregnant women with nutritional assessment and counselling, social support, and recommendations for physical activity. Ideally, a dietitian would be aware of a woman's nutrition habits pre-conception so that a nutrition plan based on pre-pregnancy BMI can be put forth that adheres to the IOM recommendations of weight gain. A dietitian could also counsel women who retain or gain excess weight post-partum by providing extra support on weight loss and additional nutritional counselling. Consideration should be given to enabling further access to dietitians as part of a multidisciplinary team for women prior to, during, and after pregnancy, as this is a critical life stage where women may be more receptive to dietary improvements and health education. It is also important that health professionals advocate for policies that aim to reduce poverty, improve health equity, and address the social determinants of health [28].

\section{Conclusion}

Many women are entering pregnancy heavier than women from previous generations. When women enter pregnancy overweight or obese, the recommendations for weight gain are difficult to adhere to and these women may gain weight excessively, increasing the likelihood of poor maternal and child health outcomes [10,12]. This increases the risk of a LGA infant, caesarean birth, and post-partum weight retention, and is associated with long-term health risks for both mother and infant, including obesity, diabetes and cardiovascular disease $[9,12]$. Taken together, this suggests that healthcare providers should counsel on the importance of appropriate weight gain, following IOM recommendations, and monitor weight gain throughout pregnancy. Addressing nutrition and exercise is imperative for low SES women who may be less informed about the importance of appropriate weight gain during pregnancy for their own and offspring's future health. Indeed, these women may also lack the skills/knowledge/support necessary to prevent eGWG [29]. Perhaps even more important than counselling on behavioural change are policies directed at socioeconomic inequality itself, since SES is in turn highly correlated with health behaviours [30]. The quantity and timing of GWG may also be important, although the exact period when eGWG negatively influences pregnancy and birth outcomes remains unclear $[10,22,23]$. Future research should therefore investigate whether there is a critical period during gestation whereby eGWG is most detrimental to maternal and child health. More attention should also be given to studying the influence of SES on GWG, so that policy interventions such as the Canadian Prenatal Nutrition Programme, Healthy Babies Healthy Children, increasing minimum wage, and affordable safe housing, can be enhanced [30].

\section{Declaration of interest}

All authors above have participated in this research, have reviewed, and agree with the content of this article. The authors report no conflicts of interest.

\section{References}

1. Government of Canada Public Health Agency of Canada. Obesity in Canada. Ottawa, Ontario: Government of Canada Public Health Agency of Canada; 2011.

2. Institute of Medicine, National Research Council. Weight gain during pregnancy: reexamining the guidelines. Washington DC: The National Academic Press; 2009.

3. Birdsong A, Byrd M, Holcomb HN, et al. Excessive gestational weight gain: long-term health risks. Int J Childbirth Educ 2014;29: $51-8$.

4. Johnson JL, Farr SL, Dietz PM, et al. Trends in gestational weight gain: the pregnancy risk assessment monitoring system, 20002009. Am J Obstet Gynecol 2015;212:806.e1-8.

5. Schack-Nielsen L, Michaelsen KF, Gamborg M, et al. Gestational weight gain in relation to offspring body mass index and obesity from infancy through adulthood. Int J Obes 2010;34:67-74.

6. McDonald SD, McKinney B, Foster G, et al. The combined effects of maternal depression and excess weight on neonatal outcomes. Int J Obes (Lond) 2015;39:1033-40.

7. Davies GAL, On K, Maxwell C, et al. Obesity in pregnancy: erSOGC Clinical Practice Gudielines, No. 239. J Obstet Gynaecol Can 2010;32:165-73.

8. Barker DJP. The Developmental Origins of adult disease. J Am Coll Nutr 2004;23:588S-5S.

9. Ashley-Martin J, Woolcott C. Gestational weight gain and postpartum weight retention in a cohort of Nova Scotian women. Matern Child Health J 2014;18:1927-35.

10. Daemers DOA, Wijnen HAA, van Limbeek EBM, et al. Patterns of gestational weight gain in healthy, low-risk pregnant women without co-morbidities. Midwifery 2013;29:535-41.

11. Savitz DA, Stein CR, Siega-Riz AM, Herring AH. Gestational weight gain and birth outcome in relation to prepregnancy body mass index and ethnicity. Ann Epidemiol 2011;21:78-85.

12. Nohr EA, Vaeth M, Baker JL, et al. Combined associations of prepregnancy body mass index and gestational weight gain with the outcome of pregnancy. Am J Clin Nutr. 2008;87:1750-9.

13. Stotland NE, Gilbert P, Bogetz A, et al. Preventing excessive weight gain in pregnancy: how do prenatal care providers approach counseling? J Womens Health (Larchmt) 2010;19:807-14.

14. Chaffee BW, Abrams B, Cohen AK, Rehkopf DH. Socioeconomic disadvantage in childhood as a predictor of excessive gestational weight gain and obesity in midlife adulthood. Emerg Themes Epidemiol 2015;12:4.

15. Tarasuk VS. Household food insecurity with hunger is associated with women's food intakes, health and household circumstances. J Nutr 2001;131:2670-6.

16. Drewnowski A, Eichelsdoerfer P. Can low-income americans afford a healthy diet? Nutr Today 2010;44:246-9.

17. Larsen K, Gilliland J. Mapping the evolution of "food deserts" in a Canadian city: supermarket accessibility in London, Ontario, 19612005. Int J Health Geogr 2008;7:16.

18. Robert SA, Reither EN. A multilevel analysis of race, community disadvantage, and body mass index among adults in the US. Soc Sci Med 2004;59:2421-34.

19. Huynh M, Borrell LN, Chambers EC. Maternal education and excessive gestational weight gain in New York city, 1999-2001: the effect of race/ethnicity and neighborhood socioeconomic status. Matern Child Health J 2014;18:138-45.

20. Gallagher A, Liu J, Probst JC, et al. Maternal obesity and gestational weight gain in rural versus urban dwelling women in South Carolina. J Rural Health 2013;29:1-11.

21. Brawarsky P, Stotland NE, Jackson Ra, et al. Pre-pregnancy and pregnancy-related factors and the risk of excessive or inadequate gestational weight gain. Int J Gynaecol Obstet 2005; 91:125-31.

22. Davenport MH, Ruchat S-M, Giroux I, et al. Timing of excessive pregnancy-related weight gain and offspring adiposity at birth. Obstet Gynecol 2013;122:255-61.

23. Drehmer M, Duncan BB, Kac G, Schmidt MI. Association of second and third trimester weight gain in pregnancy with maternal and fetal outcomes. PLoS One 2013;8:e54704.

24. Margerison-Zilko CE, Shrimali BP, Eskenazi B, et al. Trimester of maternal gestational weight gain and offspring body weight at birth and age five. Matern Child Health J 2012;16:1215-23. 
25. Muscati SK, Gray-Donald K, Koski KG. Timing of weight gain during pregnancy: promoting fetal growth and minimizing maternal weight retention. Int J Obes Relat Metab Disord 1996;20:526-32.

26. Hui A, Back L, Ludwig S, et al. Lifestyle intervention on diet and exercise reduced excessive gestational weight gain in pregnant women under a randomised controlled trial. BJOG 2012;119:70-7.

27. Shrewsbury VA, Robb KA, Power C, Wardle J. Socioeconomic differences in weight retention, weight-related attitudes and practices in postpartum women. Matern Child Health J [Internet] 2009;13:231-40.
28. Seabrook JA, Avison WR. Family structure and children's socioeconomic attainment: a Canadian sample. Can Rev Sociol 2015;52: 66-88.

29. Anderson CK, Walch TJ, Lindberg SM, et al. Excess gestational weight gain in low-income overweight and obese women: a qualitative study. J Nutr Educ Behav 2015;47: 404-11.e1.

30. Raphael D. Social determinants of health: present status, unanswered questions, and future directions. Int J Health Serv 2006;36: 651-77. 